\section{Sur une généralisation de la notion d'homéomorphie.}

Par

\section{Casimir Ku ratow ski (Lwów).}

Soient $\mathfrak{C}$ et $\mathscr{Y}$ deux espaces complets séparables et $y=f(x)$ une transformation biunivoque entre deux sous-ensembles de ces espaces. Cette transformation est dite une homéomorphie de classe $\alpha, \beta$, lorsque la fonction $f(x)$ est de classe $\alpha$ et la fonction inverse $f^{-1}(y)$ est de classe $\beta^{1}$ ).

L'homéomorphie généralisée ainsi conçue, qui comprend comme cas particulier (où $\alpha=0=\beta$ ) l'homéomorphie dans le sens habituel, s'est montrée très utile dans l'étude des ensembles boreliens (que nous supposerons toujours situés dans des espaces complets séparables).

Rappelons en d'abord quelques propriétés.

D'après un théorème de MM. Lu sin et Sou s lin, devenu classique dans la Théorie générale des fonctions, si $y=f(x)$ est une fonction binnivoque et mesurable $B$, définie sur un ensemble borelien $A$, la fonction inverse $x=f^{-1}(y)$ est aussi mesurable $B$ (sur l'ensemble $f(A)$ ). Autrement dit, chaque fonction biunivoque et mesurable $B$ (définie sur un ensemble borelien) est une homéomorphie généralisée (d'une certaine classe $\alpha, \beta$ ).

1) Voir ma Topologie I, Monografje Matematyczne t. 3, Warazawa-Lw6w 1933, p. 221. Les ensembles fermés sont dits de classe 0 multiplicative, les ensembles ouverts - de classe 0 additive. D'une façon générale, $\alpha$ étant un nombre transfini $<\Omega$, les produits (sommes) dénumbrables d'ensembles de classe $<\alpha$ sont dits do classe $\alpha$ maltiplicative (additive). Les ensembles qui sont simultanóment de classe $\alpha$ multiplicative ot additive sont dits ambigus de classe $a$.

Une fonction $f(x)$ est dite de classe a lorsque l'ensemble $f^{-1}\left(f^{\prime}\right)$, c. à d l'ensemble des $x$ tels que $f(x)$ appartient à $F$, est de classe $\alpha$ multiplicative, quel que soit l'ensemble fermé $F$.
Dans les mêmes hypothèses taites sur $f$ et $A$, l'ensemble $f(A)$ est borelien. Or, si $A$ est de classe multiplicative $\alpha>0$ et si $f$ est une homéomorphie de classe $\alpha, \beta$, l'ensemble $f(A)$ est de classe multiplicative $\left.\beta+\alpha^{1}\right)$. Il importe de remarquer que les hypothèses que l'ensemble $A$ est de classe $\alpha$ et que la fonction $f(x)$ est biunivoque de classe $\alpha$ ne suffisent pas pour calculer. la classe de l'ensemble $f(A)$ : la connaissance de la classe de la fonction inverse $f^{-1}(y)$ est indispensable. Cela tient au fait que l'on ne peut rien dire, en général, sur la classe d'une fonction inverse à une fonction de classe $\alpha$ (même à une fonction continue).

Une conséquence immédiate du théorème précédent est l'invariance de la notion de classe multiplicative $\alpha(>0)$ par rapport aux homéomorphies de classe $\alpha, 0^{2}$ ). Un cas très particulier de cet énoncé est l'invariance to pologique (c. à d. invariance par rapport aux homéomorphies de classe 0,0 ) de la classe $\alpha$, invariance qui est une conséquence du théorème de $M$. Lavrentieff sur le prolongement des fonctions continues. Ce dernier s'est montré un cas particulier du théorème suivant sur le prolongement de l'homéomorphie de classe $\alpha, \beta$ : toute homéomorphie de classe $\alpha, \beta$ entre deux ensembles arbitraires $A$ et $B$ se laisse prolonger (sans altérer sa classe) sur deux ensembles respectivement de classes multiplicatives $\alpha+\beta+1$ et $\beta+\alpha+1^{3}$ ).

Revenons sur le théorème qui permet de calculer la classe de l'ensemble $f(A)$ à l'aide de la classe de l'ensemble $A$ et de celle de l'homéomorphie $f$. Il en résulte (en remplaçant $\alpha$ par 1 et $\beta$ par $\alpha$ ) que $A$ étant un espace complet séparable et $f$ une homéomorphie de classe $0, \alpha$, l'ensemble $f(A)$ est de classe multiplicative $\alpha+1$. Comme nous allons voir $\left.{ }^{4}\right)$, ce théorème admet un théorème inverse: chaque ensemble de classe mutiplicative $\alpha+1$ se laisse obtenir d'un espace complet séparable, convenablement choisi, à l'aide d'une homéomorphie de classe $0, \alpha$. On parvient ainsi à la caractérisation

1) Topologie I, p. 222 et Comptes Rendus, Paris t. 197 (1933), p. 1090.

2) En ce qui concerne le problème de l'iuvariance de la propriété de Baire par rapport aux homéomorphies généralisées, on consultera les résaltats de M. Sierpinási publiés dans ce volume.

3) Topologie I, p. 221 et Comptes Rendas l. cit.

4) Cf. $N^{0} 3$, théor. 1. Les autres énoncés du $N^{0} 3$ rentrent dans un ordre d'idées analogue. Il est à remarquer qu'ils permettent de généraliser et de préciser plusieurs théorèmes importants de M. L a sin (voir ses Ensembles analytiques,
Paris 1930, chap. II). 
suivante des ensembles de classe multiplicative $\alpha+1$ : ces ensembles coïncident avec les ensembles qui admettent une représentation paramétrique biunivoque de classe $0, \alpha$ sur un espace complet ou encore: sur un ensemble fermé dans l'ensemble des nombres irrationnels (si $\alpha>0$ ).

Dans le cas particulièrement important où l'ensemble donné est lui-même un espace complet séparable et dense en soi, cet ensemble s'obtient par une homéomorphie de classe 0,1 de l'ensemble $\mathcal{O} \tau$ des nombres irrationnels (qui est topologiquement complet). On en déduit facilement qu'entre chaque couple d'espaces complets séparables qui ont la même puissance il existe une homéomorphie de classe $1,1^{1}$ ). Des énoncés analogues concernent les ensembles boreliens de classe arbitraire, de sorte que dans le domaine des ensembles boreliens done dans le domaine des ensembles que l'on rencontre habituellement - l'équivalence au sens de la Théorie des ensembles (identité de la puissance) se laisse réaliser toujours à l'aide d'une homéomorphie généralisée (dont la classe peut être calculée).

1. Lemme. Etant donné dans un espace métrique séparable un ensemble dense en soi non vide $D$ qui est une différence de deux ensembles fermés: $D=A-B$, il existe une suite d'ensemables fermés $F_{1}, F_{9}, \ldots$ tels que $D=F_{1}+F_{2}+\ldots$ et que la différence $D_{n}=F_{n}-\left(F_{1}+\ldots+F_{n-1}\right)$ est dense en soi et non vide, quel que soit $n$.

On peut supposer de plus que $\delta\left(F_{n}\right)<\epsilon, \delta(X)$ désignant le diamètre de l'ensemble $X$.

Démonstration. Soit $p$ un point de $D ; p$ en étant un point d'accumulation, il existe dans $D-p$ deux ensembles $U$ et $V$ tels que $\bar{U}-U=p=\bar{V}-V$ (on peut, en effet, admettre que $U$ est constitué par une suite de points $p_{n}$ tels que $\lim p_{n}=p, p_{n} \neq p, p_{n} \varepsilon D$; la définition de $V$ est analogue et, en outre, $U \cdot V=0$ ).

Supposons de plus que $\delta(U)<\epsilon$. Les ensembles $U$ et $V+B$ étant séparés (c. à d. qu'aucun d'eux ne contient de point d'accumulation de l'autre), il existe un ensemble ouvert $G$ tel que

$$
\left.U \subset G, \bar{G} \cdot(V+B)=0 \text { et } \delta(G)<\epsilon^{2}\right) \text {. }
$$

1) Le cas particulier où ces espaces sont topologiquement contenus dano l'ensemble des nombres réels a été établi par M. Sierpinaki, Fund. Math, 21 (1933), p. 66.

1) Voir par ex. Topologie I, p. $99(\mathrm{~V}, 6)$.
Posons $F_{1}=\overline{G D}$. Il vient $F_{1} \subset \bar{D} \subset A$, d'où $F_{1} \subset A-B$, car $F_{1} \cdot B C \bar{G}_{1} \cdot B=0$. L'ensemble $G$ étant ouvert, $G D$ est dense en soi et il en est de même de $F_{1}$. En outre, comme $p \varepsilon \bar{U} \subset \overline{G D}=F_{1}, p \varepsilon \bar{V}$ et $V \subset D-\bar{G} \subset D-F_{1}$, il vient $p \varepsilon F_{1} \cdot \overline{D-\bar{F}_{1}}$, ce qui prouve que l'ensemble $D-F_{1}$ n'est pas fermé.

Faisons correspondre à chaque point $x$ de $D-B_{1}$ un ensemble ouvert $H_{x}$ tel que: $x \varepsilon H_{x}, \bar{H}_{x} \cdot\left(B+F_{1}\right)=0$ et $\delta\left(H_{x}\right)<\epsilon$. En vertu du théorème de Lindelöf il existe une suite $x_{2}, x_{3}, \ldots$ telle que $D-F_{1} \subset H_{x_{1}}+H_{x_{3}}+\ldots$ Posons $F_{n}=\overline{H_{x_{n}} \cdot D}$ pour $n>1$. Il vient $F_{n} \subset \bar{D} \subset A$, d'où $F_{n} \subset D-F_{1}, \operatorname{car} F_{n} \cdot\left(B+F_{1}\right) \subset \bar{H}_{x_{n}} \cdot\left(B+F_{1}\right)=0$. Par conséquent $D=F_{1}+F_{2}+\ldots$

En outre, l'ensemble $D-F_{1}$ n'étant pas fermé, il existe une infini té d'indices $n$ tels que la différence $D_{n}=F_{n}-\left(F_{1}+\ldots+F_{n-1}\right)$ est non vide. On peut donc supposer qu'il en est ainsi de chaque $n$.

Or, $H_{x_{n}}$ étant ouvert, $H_{x_{n}} \cdot D$ est dense en soi et il en est de même de $F_{n}$. Comme ensemble ouvert dans $F_{n}$, l'ensemble $D_{n}$ est dense en soi et non vide.

Lemme généralisé. $\mathfrak{e}$ étant un espace métrique séparable et dense en soi, on peut faire correspondre à chaque système fini d'entiers positifs $n_{1}, \ldots, n_{k}$ un ensemble fermé $F_{n_{1}, \ldots, n_{k}}$ de façon que l'on ait:

$$
\begin{array}{ll}
\text { (1) } \quad F_{n_{1}, \ldots, n_{k}} \neq 0 & \text { (2) } \delta\left(F_{n_{1}, \ldots, n_{k}}\right)<1 / k \\
\text { (3) } \quad \mathfrak{Q}=\sum_{i=1}^{\infty} F_{i} & \text { (4) } D_{n_{1}, \ldots, n_{k}}=\sum_{i=1}^{\infty} F_{n_{b} \ldots, n_{k} i},
\end{array}
$$

où $D_{n_{1} \ldots, n_{k}}$ est défini par les conditions:

$$
\left\{\begin{array}{c}
D_{n_{1}, \ldots, n_{k-1,1}}=F_{n_{1}, \ldots, n_{k-1}, 1}, \\
D_{n_{1}, \ldots, n_{k-1}, t}=F_{n_{1}, \ldots, n_{k-1}, t}-\left(F_{n_{1}, \ldots, n_{k-1}, 1}+\ldots+F_{n_{1} \ldots, n_{k-1}, i-1}\right) .
\end{array}\right.
$$

Démonstration. Procédons par induction. En posant dans le lemme précédent: $D=\mathfrak{d}$ et $\epsilon=1$, on en déduit l'existence d'une suite d'ensembles fermés $F_{1}, F_{2}, \ldots$, satisfaisant aux conditions (1)-(3) pour $k=1$. En outre, les ensembles $D_{1}, D_{2}, \ldots$, sont denses en soi et non vides.

Fundamonta Mathematicae. T. XXII. 
Admettons que pour un indice $k \geqslant 1$ les ensembles $D_{n_{1} \ldots, n_{k}}$ soient denses en soi et non vides. En vertu du même lemme, il existe une suite d'ensembles fermés $F_{n_{1}, \ldots, n_{k}, 1}, F_{n_{1}, \ldots, n_{k^{2}},}, \ldots$ qui satisfont aux conditions (1) et (2) (en y remplaçant $k$ par $k+1$ ), à la condition (4), ainsi qu'à la condition supplémentaire que les ensembles $D_{n_{1}, \ldots, n_{k}, 1}, D_{n_{1}, \ldots, n_{k}, 2}, \ldots$ soient denses en soi et non vides.

Le lemme géuéralisé se trouve ainsi établi.

Remarquons que d'après (5) on a

$$
\sum_{i=1}^{\infty} D_{n_{1}, \ldots, n_{k}, l}=\sum_{i=1}^{\infty} F_{n_{i}, \ldots, n_{k}, l}
$$

On en conclut en raison de (3) et (4) que

$$
\text { (6) } \quad \mathfrak{e}=\sum_{i=1}^{\infty} D_{i} \quad \text { et } \quad \text { (7) } D_{n_{1}, \ldots, n_{k}}=\sum_{i=1}^{\infty} D_{n_{1}, \ldots, n_{k},} \text {. }
$$

On a enfin

$$
F_{n_{1}} \cdot F_{n_{1} n_{3}} \cdot F_{n_{1} n_{2} n_{\mathrm{s}}} \cdot \ldots=D_{n_{1}} \cdot D_{n_{1} n_{3}} \cdot D_{n_{1} n_{2} n_{3}} \cdot \ldots
$$

car, d'une part, $D_{n_{1} \ldots, n_{k}} \subset F_{n_{1}, \ldots, n_{k}}$ et, d'autre part, $F_{n_{1}, \ldots, n_{k}, i} \subset D_{n_{1}, \ldots, n_{k}}$ selon (4).

Thêorème. Chaque espace ae complet séparable et dense en soi se laisse obtenir de l'ensemble at des nombres irrationnels (de l'intervalle 01) par une homéomorphie de classe 0,1 ).

Démonstration. Désignons, d'une façon générale, par $\frac{1 \mid}{\mid 3_{1}}+\frac{1 \mid}{\mid 3_{2}}+\ldots$ le développement en fraction continue du nombre irrationnel $\xi \epsilon$ ə?

$\left\{F_{n_{1}, \ldots, n_{k}}\right\}$ étant le système d'ensembles fermés considéré dans le lemme généralisé, la suite $F_{3^{1}}, F_{\mathbf{3}^{1} \mathbf{z}^{\mathrm{a}}}, F_{\mathbf{3}^{1} \mathbf{3}^{3} \mathrm{z}^{\mathrm{a}}}, \ldots$ se compose, pour chaque $\mathfrak{z}$, d'ensembles fermés, non vides, déeroissants et à diamètre tendant vers 0 (cf. (1), (2) et (4)). Leur produit se réduit done à un seul point; nous le désignons par $f(3)$ :

$$
f(\xi)=F_{3^{1}} \cdot F_{3^{1} 3^{n}} \cdot F_{3^{1} z^{2} 3^{1}} \cdots
$$

1) Il serait intéressant de reconnaître si ce thérème ne se laisse pas étendre sux les ensembles $\boldsymbol{F}_{\boldsymbol{\alpha} \delta}$ composés exclusivement de points de condensation.
Ainsi, la fonction $f(\xi)$ se trouve définie sur l'ensemble $\mathfrak{d}$ tou t entier. En tenant compte de (2), on prouve facilement qu'elle est continue ${ }^{1}$.

Soit $x$ un point arbitraire de l'espace $\mathfrak{a}$. Les ensembles $D_{1}, D_{2}, \ldots$ étant disjoints (cf. (5)), on conclut de (6) qu'il existe un et un seul indice $n_{1}$ tel que $x \in D_{n_{1}}$. D'une façon analogue, si $x_{\in} D_{n_{y} \ldots, n_{k}}$, il existe selon (7) un et un seul indice $n_{k+1}$ tel que $D_{n_{1}, \ldots, n_{k}, n_{k+1}}$. Par conséquent, il existe un et un seul nombre irrationnel $z$ tel que $x \in D_{\mathfrak{z}^{1}} \cdot D_{\mathrm{z}^{1} \mathrm{z}^{\mathrm{z}}} \cdot \ldots$, c. à d. (en raisun de (8) et (9)) tel que $x=f(3)$; notamment $z=\frac{1 \mid}{\mid n_{1}}+\frac{1 \mid}{\mid n_{1}}+\ldots$

Autrement dit, la fonction $f(3)$ transforme $\partial \mathfrak{\imath}$ en l'espace $\mathfrak{d e}$ t o u t entier de façon biunivoque.

Désignons par $\mathfrak{z}=g(x)$ la fonction inverse à $x=f(\mathfrak{z})$. Il reste à démontrer que la fonction $g(x)$ est de première classe. Il suffit $\left.{ }^{4}\right)$ de démontrer qu'il en est ainsi de la fonction $z^{k}=g^{k}(x)$; c. à d. que l'entier positif $\mathfrak{z}^{k}$, considéré comme fonction de $x$, en est une fonction de première classe.

Tout revient done à démontrer que $m$ et $k$ étant deux entiers positifs arbitraires, l'ensemble $\underset{\boldsymbol{x}}{\mathrm{E}}\left[g^{k}(x)=m\right]^{3}$ ) est un $\boldsymbol{F}_{\sigma}$. Enfin cette dernière proposition sera établie dès que l'égalité suivante sera démontrée:

$$
\mathrm{E}_{x}\left[g^{k}(x)=m\right]=\sum D_{n_{1}, \ldots, n_{k-1}, m}
$$

la sommation étant étendue à tous les systèmes composés de $k-1$ entiers positifs. Car les ensembles $D_{n_{b}, \ldots, n_{k-1}, m}$ sont des $\boldsymbol{F}_{\sigma}$, comme des différences de deux ensembles fermés.

Or, posons $x=f(\xi)$. D'une part, l'égalité $g^{k}(x)=m$ donne $z^{k}=m$, d'sù $f(\mathfrak{z}) \in D_{3^{1}, \ldots, 3^{k-1}, m}$. Donc $x \in \boldsymbol{Z} D_{n_{1}, \ldots, n_{k-1}, m}$.

D'autre part, si $x \in \sum D_{n_{1}, \ldots, n_{k-1}, m}$, il vient selon (8) et (9) $x \in D_{3^{1}, \ldots, 3^{k}}, \Sigma D_{n_{1}, \ldots, n_{k-1, m}}$ et les ensembles $D_{n_{1}, \ldots, n_{k}}$ étant disjoints (pour $k$ fixe), on en tire $z^{k}=m$, c. à d. que $g^{k}(x)=m$.

1) Voir par ex. Topologie I, p. 223.

2) Ibid., p. 182

2) = ensemble des $x$ satisfaisant a la condition entre crochets [ ]. 
Corollaire. $\mathfrak{d e}_{1}$ et $\mathfrak{Q}_{2}$ étant deux espaces complets séparables de la même puissance, il existe entre eux une homéomorphie de classe $1,1$.

Démonstration. Dans le cas où les espaces considérés sont dénombrables (ou finis), le corollaire est évident, car ch aque fonction définie sur un espace dénombrable est de I-re classe.

Supposons donc que $\mathfrak{C}_{1}$ et $\mathfrak{Q}_{2}$ sont indénombrables. D'après un théorème classique, chaque espace indénombrable devient dense en soi, lorsqu'on lui enlève un ensemble clairsemé ${ }^{1}$ ) convenablement choisi. Il peut arriver que cet ensemble clairsemé soit fini (ou vide), mais alors on peut lui ajouter une suite infinie (et convergente) de points sans qu'il cesse d'être clairsemé et sans que le reste de l'espace cesse d'être dense en soi. Désignons donc par $O_{1}$ et $C_{\mathrm{a}}$ deux ensembles clairsemés infinis et tels que les ensembles $\mathfrak{a}_{1}-C_{1}$ et $a_{2}-C_{2}$ soient denses en soi. Ces deux derniers ensembles sont topologiquement complets comme des ensembles $G_{8}^{2}$ ). Il existe donc en vertu du théorème précédent deux homéomorphies $f_{1}$ et $f_{2}$ de classe 0,1 qui transforment l'ensemble $\partial$ respectivement en $\partial_{1}-C_{1}$ et $\mathfrak{a C}_{2}-C_{2} ;$ c. à d. que $f_{1}(\partial \tau)=\mathfrak{a c}_{1}-C_{1}$ et $f_{2}(\mathfrak{\partial})=\mathfrak{o C}_{2}-C_{2}$. Posons $f(x)=f_{2} f_{1}^{-1}(x)$ pour $x \in \mathfrak{Q}_{1}-C_{1}$.

La fonction $f$ est done une homéomorphie de classe 1,1 entre $\mathfrak{a}_{1}-C_{1}$ et $\mathfrak{a}_{2}-C_{2}$. Les ensembles $C_{1}$ et $C_{2}$ étant infinis et clairsemés, donc de la puissance $\aleph_{0}$, il existe une fonction biunivoque (que nous pouvons désigner encore par $f$ ) qui transforme $C_{1}$ un $C_{2}$. Cette dernière fonction étant - comme nous l'avons déjà indiqué nécessairement de I-re classe et les ensembles $C_{1}$ et $\mathfrak{Q}_{1}-C_{1}$ étant des ensembles $\boldsymbol{F}_{\sigma}$ (puisque $C_{1}$ est un $\boldsymbol{G}_{\delta}$, comme ensemble clairsemé ${ }^{\mathrm{s}}$ )), la fonction $f$ est une fonction de I-re classe sur l'espace tout entier ${ }^{4}$ ).

Il en résulte aussitôt que $f$ est une homéomorphie de classe 1,1 .

1) c. ì d. ne contenant aucun ensemble dense en soi.

2) D'aprè un théor. de M. A lexandroff, chaque ensemble $G_{\delta}$ (situé daris un enpace complet séparable) est homéomorphe a un espace complet séparable. Cf. par ex. Topologie I, p. 200.

2) D'après un théor. de M. W. H. Young. Cf. par ex. Topologie I, p. 112.

4) D'une façon plus générale, si l'espace se compose d'une suite d'ensembles de classe $\alpha$ additive sur lesquels $f$ est de classe $\alpha, f$ est sur l'espace to ut entier do classe a. Voir par ex. H. Hahn, Reelle Funktionen I, Leipzig 1932, p. 287 on Topologie I, p. 179.
Remarque. Chaque ensemble $\boldsymbol{G}_{\delta}$ (sitú́ dans un espace complet séparable) étant homéomorphe à un espace complet, le corollaire implique qu'entre chaque couple d'ensembles $G_{\delta}$ indénombrables il existe une homéomorphie de classe $1,1$.

Il serait intéressant de reconnaître ai le terme $G_{\delta}$ ne pourrait être remplacé par $\left.\boldsymbol{F}_{\alpha \delta}{ }^{1}\right)$.

Quant aux ensembles de classes supérieuree à 2, cf. le corollaire 2 da No 3.

2. Nous allons nous occuper à présent d'une opération sur les fonctions dont le rôle est ici auxiliaire mais qui présente un intérêt intrinsèque même dans le domaine de la Théorie générale des ensembles.

Considérons une suite (finie ou infinie) d'espaces $\mathfrak{a}_{1}, \mathfrak{Q}_{2}, \ldots$ et une suite de fonctions $f_{n}\left(x_{n}\right)$ définies respectivement sur ces espaces (la variable $x_{n}$ parcourant l'espace $\mathfrak{C}_{n}$ ) et dont les valeurs appartiennent à un espace fixe $\mathscr{Y}$. Soit $\mathfrak{Q}_{1} \times \mathfrak{Q}_{2} \times \ldots$ le produit cartésien des espaces $\mathfrak{Q}_{n}$, c. d̀ d. l'ensemble des suites infinies $z=\left[\boldsymbol{3}^{1}, \boldsymbol{\jmath}^{2} \ldots\right]$ telles que $\mathfrak{z}^{n} \in \mathfrak{Q}_{n}$. Désignons par $\mathfrak{z}$ l'ensemble des $\mathfrak{z}$ tels que $f_{1}\left(\mathfrak{z}^{1}\right)=$ $=f_{2}\left(\mathbf{3}^{2}\right)=f_{3}\left(3^{3}\right)=\ldots$ et définissons la fonction $f(\xi)$ pour $3 \varepsilon \mathbf{3}$ en convenant que $f(\xi)=f_{n}\left(3^{n}\right)$ quel que soit $\left.n^{2}\right)$.

La fonction $f$ jouit des propriétés suivantes:

a.

$$
f(\mathfrak{3})=f_{1}\left(\mathfrak{Q}_{1}\right) \cdot f_{2}\left(\mathfrak{Q}_{2}\right) \cdot \ldots
$$

Car la condition $y \in f(\boldsymbol{3})$ signifie qu'il existe un $\mathfrak{z}$, tel que $y=f(\mathfrak{z})$, c. à d. tel que $y=f_{n}\left(3^{n}\right)$ quel que soit $n$, donc tel que l'on ait $y \in f_{1}\left(\mathfrak{a}_{1}\right) \cdot f_{2}\left(\mathfrak{e}_{2}\right) \cdot \ldots$.

b. Si les fonctions $f_{n}$ sont biunivoques, la fonction $f$ l'esi également.

Car la condition $f(\mathfrak{z})=f\left(\xi_{1}\right)$ implique que $f_{n}\left(\mathfrak{z}^{n}\right)=f_{n}\left(\mathfrak{3}_{1}^{n}\right)$, d'où $\mathfrak{z}^{n}=\mathfrak{z}_{1}^{n}$, quel que soit $n$. Il en résulte que $\mathfrak{z}=\xi_{1}$ (puisque le $n$-ème terme de la suite $z$ est identique au $n$-ème terme de $z_{1}$ ).

c. Si $Q \subset \mathscr{Y}$, on a pour chaque $n$

$$
f^{-1}(Q)=\mathbf{3} \cdot \underset{\mathbf{3}}{\mathbf{E}}\left[f_{n}\left(\mathfrak{3}^{n}\right) \in Q\right]=\mathbf{3} \cdot \mathbf{E}_{\mathbf{3}}\left[\mathfrak{3}^{n} \in f_{n}^{-1}(Q)\right]
$$

1) (Cf. renvoi 1), p. 210.

2) En particulior, si la suite des espaces se réduit à denx termes $\mathfrak{Q}_{1}$ et $\mathfrak{Q}$, et si $\mathfrak{a}_{1}=\mathfrak{e}_{2}=\mathscr{Y}=$ l'espace des nombres réels, la fonction $F(\mathfrak{\xi})$ est une fonction réelle de variable complexe $3=x_{1}+i x_{2}$. Cette fonction est définie sur la "coarbe" $f_{1}\left(x_{1}\right)=f_{2}\left(x_{2}\right)$ située dans le plan $\mathfrak{Q}_{1} \times \mathfrak{Q}_{2}$. 
Car la condition $\mathfrak{z} \in f^{-1}(Q)$ équivaut à $f(\mathfrak{z}) \in Q$, done à l'hyputhèse que $f_{n}\left(\mathfrak{\zeta}^{n}\right) \in Q$ et que $\mathfrak{z} \in \mathbf{3}$.

d. Si les fonctions $f_{n}$ sont de classe $\alpha$, l'ensemble $\mathbf{3}$ est de classe $\alpha$ multiplicative dans le produit $\mathfrak{d}_{1} \times \mathfrak{d}_{2} \times \ldots$ et la fonction $f$ est de classe $\alpha($ sur $\left.\mathbf{3}){ }^{1}\right)$.

Par définition de $\mathfrak{z}$ on $a$, en effet,

$$
\mathbf{s}=\prod_{n=1}^{\infty} \mathbf{E}\left[f_{n}\left(\mathbf{3}^{n}\right)=f_{1}\left(\mathbf{z}^{1}\right)\right]
$$

Or les fonctions $f_{1}\left(x_{1}\right)$ et $f_{n}\left(x_{n}\right)$ étant de classe $\alpha$, l'ensemble $\underset{x_{1} x_{n}}{\mathrm{E}}\left[f_{n}\left(x_{n}\right)=f_{1}\left(x_{1}\right)\right]$ l'est également et il en est encore de même de l'ensemble $\underset{\mathfrak{z}}{\mathrm{E}}\left[f_{n}\left(\mathfrak{z}^{n}\right)=f_{1}\left(\mathfrak{z}^{1}\right)\right]$, puisque les fonctions $\mathfrak{z}^{1}$ et $\mathfrak{z}^{n}$ sont des fonctions continues de l'argument $\boldsymbol{\zeta}^{2}$ ). L'ensemble $\boldsymbol{z}$ est donc de classe $\alpha$ multiplicative.

En outre, l'égalité $f(\mathfrak{z})=f_{1}\left(\mathfrak{3}^{1}\right)$ montre que la tonction $f$ est de classe $\alpha$, comme superposition d'une fonction de classe $\alpha$ d'une fonction continue.

e. Si les fonctions $y=f_{n}\left(x_{n}\right)$ sont biunivoques et si leurs fonctions inverses $x_{n}=f_{n}^{-1}(y)$ sont de classe $\alpha$, la fonction $z=f^{-1}(y)$ est de classe $\boldsymbol{\alpha}$ (sur l'ensemble $f(\mathbf{3})$ ).

En effet, la fonction $f^{-1}(y)$ fait correspondre à chaque $y$ la suite des points $\mathfrak{z}^{1}(y)=f_{1}^{-1}(y), \mathfrak{z}^{2}(y)=f_{2}^{-1}(y), \ldots$ Ces points étant les "coordonnées" du point variable $f^{-1}(y)$ et étant des fonctions de classe $\alpha$ de la variable $y$, il en résulte ${ }^{3}$ ) que la fonction $f^{-1}(y)$ est de classe $\alpha$.

f. La propriété d'être une image de classe $0, \alpha$ d'un espace complet séparable est multiplicative au sens dénombrable.

1) Le produit cartésien $\mathfrak{Q}_{1} \times \mathfrak{a C}_{2} \times \ldots$ est métrisé par la formule $|\mathfrak{z}-y|=$
$=\sum_{i=1}^{\infty} 2^{-t} \frac{\left|\mathfrak{z}^{t}-y^{i}\right|}{1+\left|\mathfrak{z}^{t}-y^{i}\right|},\left|\mathfrak{z}^{t}-y^{i}\right|$ désignant la distance dea points $\mathfrak{z}^{t}$ et $y^{t}$ dans l'espace $\mathfrak{a C}_{l}$. Ainsi métrisé, le prodnit d'espaces complets séparables est complet séparable. Voir Topologie I, pp. 88 at 197.

2) Ibid, p. 79.

3) Ibid, p. 182
Notamment, si les espaces $\mathfrak{Q}_{n}$ sunt complets séparables et si les fonctions $f_{n}$ sont des homéomorphies de classe $0, \alpha$, la fonction $f$ est une homéomorphie de classe $0, \alpha$ (selon $\boldsymbol{d}$ et $\boldsymbol{e}$ ) transformant l'espace complet séparable $\mathfrak{z}$ (selon $\boldsymbol{d}$ où l'on pose $\alpha=0$ ) en l'ensemble $f\left(\mathfrak{Q}_{1}\right) \cdot f\left(\mathfrak{Q}_{\mathbf{2}}\right) \cdot \ldots$ (selon $\left.\boldsymbol{a}\right)$.

Remarque. On peut ajouter aux termes "complet séparable“ le terme de dimension 0 . Car les espaces $\mathfrak{a}_{n}$ étant supposés de dimension 0 , il en est de même de leur produit cartésien ${ }^{2}$ ), done de l'ensemble 3.

g. Etant données une suite d'ensembles $E_{n}$ ambigus de classe $\gamma$ dans $\mathfrak{O}_{n}$ et une suite d'homéomorphies $f_{n}$ de classe 0 , $\alpha$ et telles que $f_{n}\left(\mathfrak{Q}_{n}\right)=\mathscr{H}$, on a $f\left(\mathfrak{f}_{n}\right)=f_{n}\left(E_{n}\right)$ où l'ensemble $\mathfrak{f}_{n}=\mathbf{3} \cdot \underset{\mathbf{z}}{\mathbf{E}}\left[\mathfrak{3}^{n} \in E_{n}\right]$ est ambigu de classe $\gamma$ et $f$ est une homéomorphie de classe 0 , $\alpha$.

En effet, en posant dans $c: Q_{n}=f_{n}\left(E_{n}\right)$, il vient $E_{n}=f_{n}^{-1}\left(Q_{n}\right)$ et $f^{-1}\left(Q_{n}\right)=\mathrm{f}_{n}$. La fonction $f$ étant biunivoque (même une homéomorphie de classe $0, \alpha$ selon $\boldsymbol{d}$ et $\boldsymbol{e}$ ), on en tire: $Q_{n}=f\left(\boldsymbol{f}_{n}\right)$. Enfin $\mathfrak{3}^{n}$ étant une fonction continue de $\mathfrak{\xi}$, l'ensemble $\underset{\mathfrak{z}}{\mathrm{E}}\left[\mathfrak{\mathfrak { z }}^{n} \in E_{n}\right]$, et par conséquent $f_{n}$, est ambigu de classe $\gamma$.

3. Théorème 1. Pour qu'un ensemble $A$ situé dans un espace complet séparable soit de classe $\alpha>0$ multiplicative, il faut et il suffit qu'il se laisse obtenir par une homéomorphie de classe $0, \alpha-1^{\text {') }}$ d'un espace complet séparable. Ce dernier espace peut être supposé de dimension $\left.0,{ }^{3}\right)$ si $\alpha>1$.

Démonstration. Comme nous l'avons déjà indiqué dans l'Introduction, la condition est suffis ante.

Sa nécessité signifie, dans le cas où $\alpha=1$, que chaque ensemble $G_{\delta}$ est homéomorphe (de classe 0,0 ) à un espace complet. Ceci est un théorème connu "). Il existe, de plus, un espace complet séparable de dimension 0 et une homéomorphie de classe 0,1 qui transforme cet espace en l'espace $\boldsymbol{G}_{\delta}$ considéré ${ }^{5}$ ).

1) Ibid. p. 149 .

2) Si $\alpha$ est un nombre limite, on pose $\alpha-1=\alpha$.

3) Rappelons que chaque espace complet séparable et de dimension 0 est homéomorphe à un ensemble fermé dans l'ensemble des nombres irrationnels. Cf. par ex. Topologie I, p. 224.

4) Voir renvoi 2), p. 212.

b) Topologie I, p. 226. 
Procédons par induction. Etant donné un nombre $\alpha>1$, il est légitime d'admettre que chaque ensemble $X$ de classe $\xi<\alpha(\xi>0)$ se laisse obtenir d'un ensemble fermé dans l'ensemble $\partial \tau$ des nombres irrationnels par une homéomorphie de classe $0, \xi$.

Chaque ensemble $A$ de classe multiplicative $\alpha>1$ est de la forme $A=\prod_{n=1}^{\infty} \sum_{k=1}^{\infty} A_{n}^{k}$, les ensembles $A_{n}^{k}$ étant ambigus de classe $\alpha_{n}^{k}<\boldsymbol{\alpha}$ et étant disjoints pour $n$ fixe $\left.{ }^{1}\right)$. Par hypothèse, il existe un ensemble $B_{n}^{k}$ fermé dans $\partial$ et contenu dans l'intervalle $k, k+1$ et une homéomorphie $f_{n}^{k}$ de classe $0, \alpha_{n}^{k}$ telle que $f_{n}^{k}\left(B_{n}^{k}\right)=A_{n}^{k}$. L'ensemble $B_{n}=B_{n}^{1}+B_{n}^{2}+\ldots$ est évidemment fermé dans l'ensemble de tous les nombres irrationnels; il constitue done un espace topologiquement complet, séparable, de dimension 0 . La fonction $f_{n}$, définie sur $B_{n}$ par la condition qu'elle coïncide avec $f_{n}^{k}$ sur $B_{n}^{k}$, transforme donc $B_{n}$ en $A_{n}=\sum_{k=1}^{\infty} A_{n}^{k}$ d'une façon biunivoque et continue. De plus, la fontion $f_{n}^{-1}$ inverse à $f_{n}$ coïncide sur $A_{n}^{k}$ avec la fonction inverse à $f_{n}^{k}$ : celle-ci étant par hypothèse de classe $\alpha_{n}^{k} \leqslant \alpha-1$ et les ensembles $A_{n}^{k}$ étant ambigus de classe $\alpha_{n}^{k}$, il en résulte 2 ) que la fonction $f_{n}^{-1}$ est de classe $\alpha-1$.

Il est ainsi établi que l'ensemble $A_{n}$ s'obtient par une homéomorphie de classe $0, \alpha-1$ d'un espace complet, séparable, de dimension 0 . Il en est de même de l'ensemble $A=\prod_{n=1}^{\infty} A_{n}$ en raison de $f$.

Corollaire 1. A étant un ensemble indénombrable de classe $\alpha>1$ multiplicative, il existe un ensemble dénombrable (infini) $D$ tel que $A-D$ se laisse obtenir de l'ensemble al des nombres irrationels par une homéomorphie de classe $0, a-1$.

Car chaque espace indénombrable, complet, séparable et de dimension 0 se compose d'un ensemble dénombrable et d'un ensemble homéomorphe (de classe 0,0$)$ à $\partial{ }^{3}$ ).

Corollaire 2. $A$ et $B$ étant deux ensembles indénombrables respectivement de classes $\alpha>2$ et $\beta>2$ multiplicatives, il extste entre eux une homéomorphie de classe $\alpha-1, \beta-1$.

1) Ibid, p. 162

2) Voir renvoi \$) p. 212. p. 827.

') d'aprés un th6́or. de M. Mazurkiewicz. Voir par ex, Topologie I,
Soient, conformément au corollaire précédent, $D$ et $E$ deux ensembles dénombrables et $f_{1}$ et $f_{2}$ deux homéomorphies respectivement de classes $0, \alpha-1$ et $0, \beta-1$ et telles que $f_{1}(\mathcal{O U})=$ $=A-D, f_{\mathbf{2}}(\partial \mathfrak{Z})=B-D$. Posons $f(x)=f_{\mathbf{2}} f_{1}^{-1}(x)$ pour $x \in A-D$ et définissons $f(x)$ pour $x \in D$ de manière que l'ensemble $D$ se trouve transformé en $E$ d'une façon biunivoque.

La fonction $f$, ainsi définie, est de classe $\alpha-1$ sur $A-D$ et de classe 1 , done de classe $\alpha-1$ sur $D$. Les ensembles $D$ et $A-D$ étant des ensembles $G_{\delta \sigma}$ par rapport à $A$ (donc des ensembles de classe $\alpha-1$ additive), la fonction $f$ est de classe $\alpha-1$ sur l'ensemble $A$ tout entier ${ }^{1}$ ).

Par raison de symétrie $f^{-1}$ est de classe $\beta-1$ sur $B$.

Théorème 2. Etant donné dans un espace complet séparable $\mathscr{Y}$ un ensemble $A$ ambigu de classe $\alpha+\beta$, avec $\alpha>0$, l'espace $\mathscr{Y}$ se laisse obtenir d'un espace complet, séparable, de dimension 0 , par une homéomorphie de classe $0, \alpha$ qui transforme un ensemble ambigu de classe $\beta$ en $A^{2}$ ).

Démonstration. Procédons par induction.

1) Si $\beta=0$, il existe d'après le théor. 1 deux ensembles $B_{1}$ et $B_{2}$ fermés dans $\partial \mathcal{O}$ et situés respectivement dans les intervalles 0,1 et 1,2 et deux homéomorphies $f_{1}$ et $f_{2}$ de classe $0, \alpha$ telles que $f_{1}\left(B_{1}\right)=A$ et $f_{2}\left(B_{2}\right)=\mathscr{Y}-A$ (car $A$ et $\mathscr{Y}-A$ sont de classe multiplicative $\alpha$ ). On définit la fonction $f$ sur l'ensemble topologiquement complet $B=B_{1}+B_{2}$, en convenant qu'elle coïncide respectivement avec $f_{1}$ et $f_{2}$ sur $B_{1}$ et $B_{2}$. La fonction $f$ est donc biunivoque, continue et $f(B)=\mathscr{Y}$. En outre, comme la tonction $f^{-1}$ coïncide respectivement sur les ensembles $A$ et $\mathscr{Y}-A$ avec $f_{1}^{-1}$ et $f_{2}^{-1}$, qui sont de classe $\alpha$, de même que ces ensembles, la fonction $f^{-1}$ est aussi de classe $\alpha$. La fonction $f$ est done une homéomorphie de classe $0, \alpha$. Enfin $A=f\left(B_{1}\right)$ et $B_{1}$ est évidemment ambigu de classe 0 dans $B$ (c. à d. que $B_{1}$ y est fermé et ouvert).

2) Si $\beta=\gamma+1$, l'ensemble $A$ est la limite d'une suite d'ensembles $A_{n}$ ambigus de classe $\left.\alpha+\gamma^{3}\right)$ :

$$
A=\prod_{n=0}^{\infty} \sum_{k=0}^{\infty} A_{n+k}=\sum_{k=0}^{\infty} \prod_{n=0}^{\infty} A_{n+k}
$$

2) Voir renvoi *) p. 212.

2) Pour le cas $\beta=1$, voir N. La ain, loc. cit,

2) Yoir p. ex. Topologie I, p. 116. 
Par hypothèse il existe: une suite d'espaces $\mathfrak{Q}_{n}$ complets, séparables, de dimension 0 , une suite d'ensembles $E_{n}$ ambigus de classe $\gamma$ dans $\mathfrak{Q}_{n}$ et une suite d'homéomorphies $f_{n}$ de classe $0, \alpha$ telles que $f_{n}\left(\mathfrak{Q}_{n}\right)=\mathscr{Y}$ et $f_{n}\left(E_{n}\right)=A_{n}$. D'après $g$ il existe un espace $\mathfrak{z}$ complet, séparable, de dimension 0 , une suite d'ensembles $f_{n}$ ambigus de classe $\gamma$ dans $\mathfrak{3}$ et une homécmorphie $f$ de classe $0, \alpha$ telle que $f(\boldsymbol{3})=\mathscr{Y}$ et $f\left(\mathfrak{f}_{n}\right)=A_{n}$.

La fonction $f$ étant biunivoque, il vient

$$
\begin{aligned}
A & =\prod_{n=0}^{\infty} \sum_{k=0}^{\infty} f\left(\mathrm{f}_{n+k}\right)=f\left\{\prod_{n=0}^{\infty} \sum_{k=0}^{\infty} \mathrm{f}_{n+k}\right\} \\
& =\sum_{k=0}^{\infty} \prod_{n=0}^{\infty} f\left(\mathrm{f}_{n+k}\right)=f\left\{\sum_{k=0}^{\infty} \prod_{n=0}^{\infty} \mathrm{f}_{n+k}\right\} .
\end{aligned}
$$

Ainsi $A$ s'obtient à l'aide de la fonction $f$ de la limite des ensembles $f_{n}$ et celle-ci est un ensemble ambigu de classe $\beta=\gamma+1$, comme limite d'ensembles ambigus de classe $\gamma$.

3) $\mathrm{Si} \beta$ est un nombre limite, on a

$$
A=\prod_{n=1}^{\infty} A_{2 n} \quad \text { et } \quad \mathscr{Y}-A=\prod_{n=1}^{\infty} A_{2 n-1}
$$

où $A_{n}$ est ambigu de classe $\alpha+\gamma_{n}$ avec $\gamma_{n}<\beta$.

Par hypothèse il existe, comme auparavant, une suite d'espaces $\mathfrak{Q}_{n}$ complets, séparables, de dimension 0 , une suite d'ensembles $E_{n}$ ambigus de classe $\gamma_{n}$ dans $\mathfrak{Q C}_{n}$ et une suite d'homéomorphies $f_{n}$ de classe $0, \alpha$ telles que $f_{n}\left(\mathfrak{Q}_{n}\right)=\mathscr{Y}$ et que $f_{n}\left(E_{n}\right)=A_{n}$. D'après $\boldsymbol{g}$ il existe un espace $\mathbf{3}$ complet, séparable, de dimension 0 , une suite d'ensembles $\mathfrak{f}_{n}$ ambigus de classe $\gamma_{n}$ dans $\mathfrak{z}$ et une homéomorphie $f$ de classe $0, \alpha$ telle que $f(\mathbf{5})=\mathscr{Y}$ et $f\left(\mathfrak{f}_{n}\right)=A_{n}$.

La fonction $f$ étant biunivoque, il vient

$$
A=\prod_{n=1}^{\infty} f\left(\mathrm{f}_{2 n}\right)=f\left\{\prod_{n=1}^{\infty} \mathrm{f}_{2 n}\right\} \quad \text { et } \quad \mathscr{S}-A=f\left\{\prod_{n=1}^{\infty} \mathrm{f}_{2 n-1}\right\} \text {, }
$$

d'où on conclut que $\prod_{n=1}^{\infty} \mathrm{f}_{\mathrm{sn-1}}=\mathbf{3}-\prod_{n=1}^{\infty} \mathrm{f}_{2 n}$ (car $\mathscr{Y}=f(\mathbf{3})$ ).

L'ensemble $\prod_{n=1}^{\infty} f_{2 n}$ est donc ambigu de classe $\beta$, comme ensemble qui est - de même que son complémentaire - de classe $\beta$ multiplicative.

Theorème 3. Le théorème précédent reste vrai, lorsqu'on y remplace les classes ambigues par les classes multiplicatives.

En effet, tout ensemble $A$ de classe multiplicative $\alpha+\beta>0$ est de la forme $A=A_{1} \cdot A_{2} \cdot \ldots$ où les ensembles $A_{n}$ sont ambigus de classe $\alpha+\beta$. Il existe donc selon le théor. 2 une suite d'espaces $\mathfrak{C C}_{n}$ complets, séparables, de dimension 0 , une suite d'ensembles $E_{n}$ ambigus de classe $\beta$ et une suite d'homéomorphies $f_{n}$ de classe $0, \alpha$ telles que $f_{n}\left(\mathfrak{Q}_{n}\right)=\mathscr{Y}$ et $f_{n}\left(E_{n}\right)=A_{n}$. D'aprés $g$ il existe un espace 5 complet, séparable, de dimension 0 , une suite d'ensembles $f_{n}$ ambigus de classe $\beta$ dans $\boldsymbol{Z}$ et une homéomorphie $f$ de classe $0, \alpha$ telle que $f(\mathbf{z})=\mathscr{P}$ et $f\left(\mathfrak{f}_{n}\right)=A_{n}$.

La fonction $f$ étant biunivoque, il vient

$$
A=f\left(\mathfrak{f}_{1}\right) \cdot f\left(\mathfrak{f}_{2}\right) \cdot \ldots=f\left(\mathrm{f}_{1} \cdot \mathrm{f}_{2} \cdot \ldots\right) .
$$

L'ensemble $f_{1} \cdot f_{2} \cdot \ldots$ étant de classe $\beta$ multiplicative, le théorème se trouve démontré.

4. Theorème (d'existence). A chaque couple de nombres $\alpha, \beta$ (inférieurs $\grave{a} \Omega$ ) correspond une transformation $f$ de l'ensemble des

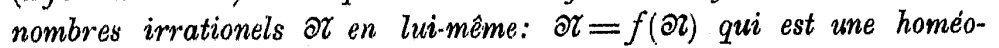
morphie précisément de classe $\alpha, \beta$ (c. $a \grave{a} d$. que ni la fonction $f$ $n^{\prime}$ est de classe $<\alpha$, ni la fonction $f^{-1} n^{\prime}$ est de classe $\left.<\beta\right)$.

Démonatration. Considérons d'abord le cas où $\beta=0$.

L'existence d'une homéomorphie de classe 1, 0 qui n'est pas une homéomorphie dans le sens habituel étant triviale, admettons que $\alpha>1$.

Soit $A$ un sous-ensemble de $\partial \tau$ ambigu de classe $\alpha$ et qui ne se laisse pas décomposer en un ensemble dénombrable et un ensemble de classe multiplicative $\left\langle\boldsymbol{\alpha}^{1}\right.$ ). D'après le théor. 2 il existe une homéomorphie $f$ de classe $\alpha, 0$ telle que $f(\partial \boldsymbol{d})$ est fermé dans $\partial$

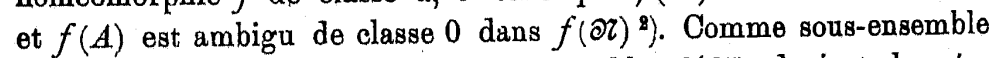
fermé et indénombrable de $\mathscr{\partial}$, l'ensemble $f(\mathfrak{\partial})$ devient homéo-

1) Cette dernière hypothèse signifie, quand $a>2$, que $A$ n'est pas de classe multiplicative $<\alpha$; pour $\alpha=2$ elle signifie que $A$ (qui est an $F_{\sigma \delta}$ et $G_{\delta \sigma}$ ) n'est pas somme d'un ensemble $G_{\delta}$ et d'un ensemble dénombrable. L'existence des ensembles $A$ de ce genre se démontre facilement.

2) Notamment l'homéomorphie $f$ est inverse à celle dn théor. 2. En outre $\mathscr{Y}=\partial t, \beta=0$. 
morphe à $\partial \mathfrak{\imath}$ en supprimant un ensemble dérombrable convenablement choisi. Autrement dit, il existe un ensemble dénombrable $D$ tel que l'ensemble $N_{1}=f(\mathfrak{d}-D)$ est homéomorphe à $\partial \mathcal{~}$.

L'ensemble $D$ étant dénombrable, la différence $N_{2}=\partial \tau-D$ est évidemment homéomorphe à $\partial \tau$.

Pour établir notre théorème (dans le cas $a, 0$ ), il suffit donc de démontrer que la fonction $f$ n'est pas de classe $\xi<\alpha$ sur l'ensemble $N_{2}$.

Supposons que $f$ soit de classe $\xi>0$ sur $N_{2}$. Par conséquent, $A N_{2}$ est de classe multiplicative $\xi$ par rapport à $N_{2}$ (puisque l'ensemble $f\left(A N_{z}\right)=f(A) \cdot N_{1}$ est fermé dans $\left.N_{1}\right)$. Autrement dit, il existe un ensemble $B$ de classe multiplicative $\xi$ (dans $a t$ ) tel que $B N_{\mathrm{q}}=A N_{\mathrm{q}}=A-D$, d'où $A=B N_{2}+A D$. Or, l'ensemble $N_{2}$ étant de classe multiplicative $\xi$ (comme ensemble $G_{\delta}$ ), l'ensemble $B N_{2}$ l'est également. L'ensemble $A D$ étant dénombrable, on en conclut que $\alpha \leqslant \xi$.

Ceci établi, passons au cas général où $\alpha$ et $\beta$ sont arbitraires. Soient $f_{1}$ et $f_{2}$ deux homéomorphies respectivement de classe $\alpha, 0$ et $0, \beta$ telles que $f_{1}\left(N^{1}\right)=N^{1}$ et $f_{2}\left(N^{2}\right)=N^{2}$, où $N^{1}$ et $N^{2}$ désignent respectivement les parties de or contenus dans les intervalles $0,1 / 2$ et $1 / 2,1$. En convenant que la fonction $f$ coïncide sur $N^{1}$ avec $f_{1}$ et sur $N^{2}$ avec $f_{2}$, on définit une homéomorphie de classe $\alpha, \beta$ telle que $f(\mathfrak{O})=\partial$ t. Les homéomorphies $f_{1}$ et $f_{2}$ étant supposées précisément des classes $\alpha, 0$ et $0, \beta, f$ est précisément de classe $\alpha, \beta$.

\section{Sur les points de densité au sens fort.}

Par

\author{
Fréd éric Riesz (Szeged, Hongrie).
}

1. Dans son livre sur l'intégration récemment paru $\left.{ }^{1}\right)$, M. S a k s vient d'établir le théorème suivant.

Presque tous les points d'un ensemble plan en sont des points de densité au sens fort.

L'ensemble en question est supposé d'être envisagé après avoir fixé un système orthogonal de courdonnées $x, y$ et ce que le théorème affirme, c'est que, excepté au plus certains points dont l'ensemble est de mesure nulle, tous les autres points $X$ de l'ensemble $E$ envisagé jouissent de la propriété suivante: pour toute suite indéfinie de rectangles $R_{n}$ parallèles aux axes, comprenant le point $X$ et dont les diagonales deviennent infiniment petites avec $1 / n$, la densité moyenne de $E$ par rapport à $R_{n}$ (c'est la mesure extérieure de la partie de $E$ comprise dans $R_{n}$ divisée par l'aire de $R_{n}$ ), a pour limite l'unité.

Ce théorème comporte une généralisation surprenante du célèbre théorème de M. Lebesgue sur les points de densité; en effet on était forcé jusqu'à ces derniers jours, par des raisons de méthode, à n'admettre que des suites $R_{n}$ dites régulières, savoir telles que le rapport des cotés adjacents reste entre des bornes finis indépendants de $n$.

M. Saks déduit son résultat d'un théorème de M. St e pa n off sur les différentielles approximatives, théorème dont la démonstration n'est pas des faciles. Vu limportance du théorème de M. Saks

1) 8. S a ka: Théorie do l'intégrale, Monografje Matematyczne, t. II, Wargzawa 1933, p. 231. 